\title{
Desempenho acadêmico nas séries do ensino fundamental: Relação com o desenvolvimento social'
}

\author{
Academic performance in basic education grades: Relation with social development
}

Fabiana Cia ${ }^{[a]}$, Carolina Severino Lopes da Costa ${ }^{[b]}$

\footnotetext{
${ }^{[a]}$ Professora adjunta do Curso de Licenciatura em Educação Especial da Universidade Federal de São Carlos, São Carlos (UFSCar), São Paulo, SP - Brasil, e-mail: fabianacia@hotmail.com

${ }^{[b]}$ Psicóloga, Mestre e Doutora em Educação Especial, Programa de Pós-Graduação em Educação Especial, Universidade Federal de São Carlos, São Carlos (UFSCar), São Paulo, SP - Brasil, e-mail: carollina_costa@yahoo.com.br
}

Recebido: 01/03/2011 Received: 03/01/2011 Approved: 06/29/20111

\section{Resumo}

Este estudo teve por objetivos: (a) verificar a relação entre os problemas de comportamento, o repertório de habilidades sociais e o desempenho acadêmico de crianças e adolescentes e (b) identificar a porcentagem de crianças e adolescentes com problemas de comportamento e baixo desempenho acadêmico, segundo a opinião dos professores. Participaram do estudo 27 professores, de um total de 215 alunos da $1^{\text {a }}$ a $8^{\underline{a}}$ série do ensino fundamental, com média de idade de nove anos. As professoras responderam ao Social Skills Rating System - Versão para Professores (que avalia o repertório de habilidades sociais, os problemas de comportamento e o desempenho acadêmico do aluno). Utilizou-se o teste de correlação de Pearson para verificar a relação entre as variáveis estudadas. Os resultados mostraram que o repertório de habilidades sociais e os problemas de comportamento (internalizantes, externalizantes e total) estavam negativamente correlacionados entre si, ou seja, quanto maior o repertório de habilidades, menor os índices de problemas de comportamento. Além disso, quanto maior os problemas de comportamento, menor o desempenho acadêmico das crianças e dos adolescentes. Respondendo ao segundo objetivo, mais da metade das crianças e dos adolescentes foram avaliados pelas professoras com baixo repertório de habilidades sociais e com altos índices de problemas de comportamento e $27,3 \%$ da amostra apresentou desempenho acadêmico abaixo da média. Esses resultados apontam para a necessidade de intervir com crianças e adolescentes para melhorar o repertório de habilidades sociais e assim diminuir a probabilidade de apresentar problemas ao longo do desenvolvimento.

Palavras-chave: Habilidades sociais. Problemas de comportamento. Desempenho acadêmico. Fatores de risco.

\section{Abstract}

This study aimed to: (a) examine the relationship between behavioral problems, the repertoire of social skills and academic performance among children and adolescents and (b) identify the percentage of children and adolescents with behavioral problems and low academic performance,

1 Trabalho apresentado no IV Congresso Brasileiro de Educação Especial (modalidade trabalho completo) 
according to the opinion of teachers. The study included 27 teachers, a total of 215 students from 1 st to 8 th grade of elementary school, with nine years old average age. The teachers answered the Social Skills Rating System - Teacher version (which assesses the repertoire of social skills, behavioral problems and academic performance of students). We used the Pearson correlation test to investigate the relation between the variables being studied. The results showed that the repertoire of social skills and behavior problems (internalizing, externalizing and total) were negatively correlated, the larger the repertoire of skills, the lower the rates of behavior problems. Moreover, the higher the behavioral problems, the lower the academic performance of children and adolescents. Responding to the second goal, over half of children and adolescents were evaluated by teachers having low social skills repertoire and high rates of behavioral problems and $27.3 \%$ of the sample had below-average academic performance. These results point to the need to intervene with children and adolescents to enhance the repertoire of social skills and, thus, decrease the probability of having problems along the development.

Keywords: Social skills. Behavior problems. Academic performance. Risk factors.

\section{Introdução}

Sabe-se que o ingresso na escola demanda da criança a aquisição e fluência de desempenhos novos e variados como seguir regras, fazer e responder perguntas, fazer e recusar pedidos, pedir informações, evitar confrontos com colegas belicosos, lidar com zombarias de colegas, discriminar momento oportuno de conversar, de brincar, de prestar atenção às explicações fornecidas pelos educadores, fazer e manter amizades (Del Prette \& Del Prette, 1999); todos esses desempenhos são considerados como classes e/ou subclasses de habilidades sociais. Tais desempenhos têm uma exigência crescente, ao longo dos anos do ensino fundamental.

Identificar o repertório de habilidades sociais das crianças torna-se um fator importante na área de educação especial, considerando que a maioria das crianças que é encaminhada para serviços de educação especial apresenta dificuldades de aprendizagem e/ou problemas de comportamento. Por exemplo, no ano de 2006, crianças com condutas típicas "dificuldades causadas por comportamentos que tendem a prejudicar e, por vezes inviabilizar as relações do aluno com seu professor e/ou com seus colegas, com materiais de uso pessoal e coletivo e, ainda, o processo de ensino aprendizagem" (Brasil, 2002, p. 15), ocupavam o segundo lugar no número de matrículas em serviços de educação especial (Brasil, 2006). Somado a isso, vários estudos apontam para a existência de correlação entre habilidades sociais, problemas de comportamento e desempenho acadêmico (D'Avila-Bacarji, Marturano \& Elias, 2005; Del Prette \& Del Prette, 2005; Dessen \& Szelbraciowski, 2004; Dunn, Cheng, O'Connor \& Bridges, 2004; Vila, 2005).

Em pesquisa nacional sobre variáveis sócio-demográficas associadas à ocorrência de comportamentos problemáticos e suas relações com as habilidades sociais e dificuldades acadêmicas, Bandeira, Rocha, Souza, Del Prette e Del Prette (2006) realizaram uma pesquisa com 257 estudantes de $1^{\text {a }}$ a $4^{\text {a }}$ séries do ensino fundamental, 185 pais (informantes) e 12 professores. Para a coleta de dados foi aplicado o questionário Critério Brasil e a escala Social Skills Rating System (SSRS). Os resultados mostraram que, na classificação feita pelos professores, 6,65\% das crianças apresentaram muitos comportamentos problemáticos e 9,37\% apresentaram poucos comportamentos problemáticos. Adicionalmente, a ocorrência de tais problemas foi mais elevada em crianças do sexo masculino, de nível socioeconômico mais baixo (maior frequência em escolas públicas que em escolas particulares) e com desempenho acadêmico mais deficitário.

De acordo com Del Prette e Del Prette (2001), o termo habilidades sociais refere-se ao conjunto de classes e subclasses comportamentais presentes no repertório do indivíduo que visa atender às diversas demandas das situações interpessoais. Entretanto, uma pessoa pode ter um amplo repertório de habilidades sociais, mas não ser capaz de 
articulá-lo de modo a atingir seus objetivos na interação social. Quando se avalia um repertório de habilidades sociais, é importante avaliar déficits e excessos comportamentais (Del Prette \& Del Prette, 2005).

Os déficits em habilidades sociais normalmente estão associados à presença de comportamentos problemáticos no repertório do indivíduo. Os problemas de comportamento prejudicam as relações interpessoais em sala de aula pela rejeição por pares e adultos (Kauffman, 2001; Walker \& Sprague, 1999) e, normalmente, são acompanhados pelo baixo desempenho acadêmico e por queixas escolares (Brancalhone, Fogo \& Williams, 2004; Kauffman, 2001; Marinho, 2003; Marturano, Toller \& Elias, 2005; Santos \& Graminha, 2006).

De acordo com a psicopatologia do desenvolvimento infantil (Achenbach, 1991; Achenbach \& Edelbrock, 1978; Del Prette \& Del Prette, 2005; Hinshaw, 1992; Kazdin, 1987, 1996; Pacheco, Alvarenga, Reppold, Piccinini, \& Hutz, 2005), os problemas emocionais e comportamentais são classificados em dois grandes grupos: os problemas emocionais e comportamentais externalizantes e internalizantes.

Os problemas emocionais e comportamentais externalizantes referem-se a ações dirigidas a outras pessoas e sua manifestação é observada em transtornos que envolvem agressividade física e/ou verbal, comportamentos desafiantes, antissociais (mentir, roubar) e, ainda, risco de uso de substâncias psicoativas. Os problemas internalizantes referem-se a comportamentos, pensamentos e sentimentos voltados para o próprio indivíduo e estão associados, de modo mais frequente, a transtornos como fobia social, ansiedade, depressão, isolamento social, baixa autoestima, entre outros (Del Prette \& Del Prette, 2005).

A literatura aponta para diversas variáveis relacionadas aos problemas de comportamento de crianças e adolescentes. Dentro das variáveis individuais observam-se quatro subcategorias:

a) temperamento da criança, que se refere à dimensão de criança fácil/difícil, humor com tendência a negatividade, menores níveis de tolerância a novos estímulos, menor adaptabilidade a mudanças;

b) dificuldades e déficits neuropsicológicos, como os identificados em várias funções como linguagem, memória, coordenação motora, integração dos sentidos visão e audição e em funções "executivas" do cérebro; c) níveis subclínicos de Transtorno de Conduta, sinais precoces de agressividade e dificuldade de manejo da criança, comportamentos antissociais, observados desde a pré-escola em diferentes contextos (casa, escola e comunidade);

d) performance intelectual e acadêmica, caracterizada por deficiências acadêmicas e baixos níveis de funcionamento intelectual. Além disso, há ainda algumas características como atribuição de intenção hostil aos demais, ressentimento e desconfiança (Kazdin, 1996; Graminha, 1998; Patterson, Debaryshe \& Ramsey, 1989).

Para González (2007), as condutas são consequências da aprendizagem ocorrida no meio familiar, escolar, social e comunitário onde as pessoas vivem. Desse modo, pesquisas mostram que os problemas de comportamento estão também relacionados diretamente às variáveis familiares, em que as crianças cujos pais falham nos cuidados/ práticas parentais (práticas parentais inadequadas, disciplina inconsistente, nível de organização e estabilidade no lar) e no suporte escolar estão em risco de desenvolver problemas de comportamento e comportamentos desviantes na adolescência (Ablow, Measelle, Cowan \& Cowan, 2009; Dessen \& Costa, 2005; Gomide, 2004; Kazdin \& Whitley, 2003; Lindsey, Caldera \& Iankersley, 2009; Marturano, Linhares \& Loureiro, 2004; Parke, 2004). Crianças que sofrem de maus-tratos, abuso ou problemas de formação de apego com os pais também apresentam maior risco de desenvolver problemas comportamentais (Lopes, 2004).

Há também características ambientais/contextuais mais amplas associadas a ocorrência de problemas de comportamento como moradia pobre, baixo nível socioeconômico, maiores níveis de estresse parental e poucas condições para enfrentamento das situações de estresse, problemas financeiros dos pais (dívidas de jogo, dívidas em bancos), morar em bairros com vizinhança perigosa, problemas psiquiátricos e/ou doenças de um dos membros da família, desemprego, que prejudicam a interação pais-filhos (Kazdin, 1996; Patterson, et al., 1989; Patterson, Reid \& Dishion, 1992).

Mas há, ainda, características específicas do contexto escolar consideradas como fatores de risco à ocorrência e/ou manutenção de problemas de 
comportamento dos alunos (não físico, mas das relações interpessoais), como pouca ênfase do professor em trabalhos escolares, pouco tempo gasto pelo professor nas lições, pouca ênfase sobre a responsabilidade individual dos estudantes, pobres condições de trabalho (recursos físicos), baixa expectativa do professor com relação ao aluno, pouca atenção do professor aos problemas do aluno, depreciação (por outras crianças da escola, vizinhança ou bairro), rejeição e agressão (também por parte de pares), inadequação da professora (Graminha, 1998, p. 76), greve escolar e mudança de professora/classe/escola.

Se por um lado há características do contexto escolar que podem favorecer a ocorrência de comportamentos problemáticos, por outro, verifica-se que a permanência na escola pode configurar-se como um fator de proteção durante a adolescência. Uma pesquisa realizada por Gallo e Williams (2008) identificou que a permanência na escola, na adolescência, é um fator de proteção para desenvolvimento e manutenção de comportamentos infracionais e que o abandono à escola estava associado a uma maior gravidade de atos infracionais, incluindo a utilização de armas e uso de substâncias psicoativas. Considera-se, portanto, relevante identificar o repertório de habilidades sociais, problemas de comportamento e desempenho acadêmico de crianças do ensino fundamental, uma vez que tais crianças estão em um período de transição da infância para a adolescência.

Considerando a importância do repertório de habilidades sociais para o desenvolvimento infantil e da correlação entre o desempenho acadêmico e a competência social de escolares, torna-se oportuno investigar a existência de tais correlações ao longo do ensino fundamental. Assim, esse estudo teve por objetivos: (a) identificar a porcentagem de crianças e adolescentes com problemas de comportamento e baixo desempenho acadêmico, segundo a opinião de professores e (b) verificar a relação entre os problemas de comportamento, o repertório de habilidades sociais e o desempenho acadêmico de crianças e adolescentes.

\section{Método}

\section{Participantes}

Participaram desse estudo 27 professoras. Dentre esse contingente, seis delas estavam cursando o terceiro grau, tendo apenas a formação de magistério e o restante das professoras tinham $3^{\circ}$ grau completo (17 formadas em Pedagogia, uma em Letras e outra em Pedagogia e Letras, em Educação Física e em Ciências Sociais). Além disso, uma professora tinha o título de mestre e duas estavam cursando o doutorado.

Essas professoras avaliaram um total de 215 crianças, sendo $49,3 \%$ do sexo masculino e $50,7 \%$ do sexo feminino, com média de idade de nove anos, variando entre seis e 14 anos. Quanto à série em que estudavam, $14,0 \%$ estava na $1^{\text {a }}$ série, ${ }^{2} 44,9 \%$ na $2^{\text {a }}$ série, $09,3 \%$ na 3 a série, $11,6 \%$ na $4^{\text {a }}$ série, $09,6 \%$ na $5^{\underline{a}}$ série, $10,7 \%$ na $6^{\underline{a}}$ série, $01,9 \%$ na $7^{a}$ série e $0,9 \%$ na $8^{\text {a }}$ série do ensino fundamental.

\section{Local de coleta de dados}

A coleta de dados junto aos participantes (professores) ocorreu em três escolas públicas e em uma Organização Não Governamental, situadas em uma cidade de médio porte no Estado de São Paulo. Tal instituição oferece atividades de complementação à escola regular, como apoio pedagógico, informática, esportes, dança, música, artesanato, horta, jardinagem, culinária, entre outras. Atende 120 crianças e adolescentes de baixo poder aquisitivo, com idades variando entre 7 e 14 anos.

Dentre as três escolas públicas, duas eram municipais, sendo uma localizada em um bairro de classe socioeconômica baixa, com aproximadamente 413 alunos, distribuídos entre a $1^{\text {a }}$ e a $4^{\text {a }}$ série, e a outra localizada em um bairro de classe socioeconômica baixa e média baixa, cujos moradores trabalhavam, em grande parte, em uma empresa da cidade, com aproximadamente 946 alunos distribuídos entre a $1^{\text {a }}$ e a $8^{\text {a }}$ séries. Uma das escolas era estadual, localizada na região central, com aproximadamente 800 alunos, distribuídos entre a $1^{\mathrm{a}}$ e a $8^{\mathrm{a}}$ série.

\footnotetext{
2 Utilizou-se o termo série ao invés de ano, pois no período da coleta de dados as escolas ainda estavam utilizando a primeira terminologia.
} 


\section{Instrumentos}

\section{Social Skills Rating System - SSRS - Versão para Professores}

Esta versão foi aplicada para avaliar a percepção das professoras quanto ao repertório de habilidades sociais, a existência e intensidade de problemas de comportamento internalizantes e externalizantes e o desempenho acadêmico das crianças e dos adolescentes, em contexto de sala de aula, sendo composta por três escalas tipo Likert:

a) 30 itens, em que a professora assinala qual a frequência (nunca, algumas vezes e muito frequente) que o aluno emite cada uma das respostas nas diferentes situações de interação social (sendo distribuídos esses itens em cinco fatores: Responsabilidade/Cooperação, Asserção Positiva, Autocontrole, Autodefesa e Cooperação com Pares);

b) 18 itens em que a professora assinala qual a frequência (nunca, algumas vezes e muito frequente) que o aluno emite cada um dos comportamentos problema (sendo distribuídos esses itens em dois fatores: comportamentos problemáticos externalizantes e comportamentos problemáticos internalizantes);

c) nove itens em que a professora avalia o desempenho acadêmico do aluno (Gresham \& Elliott, 1990, validado para o nosso contexto por Bandeira, Del Prette, Del Prette \& Magalhães, 2009). A análise de consistência interna mostrou um $\alpha=0,81$.

\section{Procedimento de coleta de dados}

Inicialmente, as pesquisadoras entraram em contato com as professoras, para explicar os objetivos da pesquisa e como seria a coleta de dados. Em seguida, com as professoras que quiseram participar, foi solicitado que assinassem o Termo de Consentimento Livre e Esclarecido. Em outro momento, a pesquisadora forneceu as informações de como preencher os instrumentos e esclareceu as dúvidas das professoras relativas a estes instrumentos, se dirigindo à sala de aula e fornecendo as informações individualmente. Assim, as professoras puderam preencher os instrumentos em um local de sua preferência. A coleta de dados com as professoras foi realizada de modo a não prejudicar suas atividades profissionais.

\section{Procedimento de análise de dados}

Os dados referentes ao SSRS-Versão para professores foram analisados de acordo com o manual do mesmo. Para correlacionar o repertório de habilidades sociais, os problemas de comportamento e o desempenho acadêmico dos alunos, utilizou-se o Teste de Correlação de Pearson (SPSS-For Windows).

\section{Resultados e discussão}

A Tabela 1 mostra os escores obtidos por meio do SSRS-Versão para professores, quanto ao repertório de habilidades sociais, aos problemas de comportamento externalizantes, aos problemas de comportamento internalizantes e ao desempenho acadêmico das crianças e dos adolescentes.

Tabela 1 - Repertório de habilidades sociais, problemas de comportamento externalizantes, problemas de comportamento internalizantes e desempenho acadêmico das crianças e dos adolescentes, segundo avaliaç̃o das professoras

\begin{tabular}{lccc}
\hline Habilidades sociais & Média & D. P. & Escore médio \\
\hline $\begin{array}{l}\text { F1-Responsabilidade/ } \\
\text { Cooperação. }\end{array}$ & 19,6 & 06,7 & $18,12-28,54$ \\
\hline $\begin{array}{l}\text { F2-Asserção positiva. } \\
\text { F3-Autocontrole. }\end{array}$ & 10,1 & 04,0 & $10,44-17,40$ \\
\hline F4-Autodefesa. & 03,0 & 04,3 & $11,95-17,53$ \\
\hline F5-Cooperação com pares. & 04,4 & 02,1 & $2,79-6,15$ \\
\hline $\begin{array}{l}\text { Habilidades sociais total } \\
\text { Problemas de comporta- }\end{array}$ & 31,2 & 15,8 & $37,63-58,7$ \\
$\begin{array}{l}\text { mento externalizantes } \\
\text { Problemas de comporta- } \\
\text { mento internalizantes }\end{array}$ & 04,0 & 05,7 & $3,70-8,31$ \\
\hline $\begin{array}{l}\text { Problemas de compor- } \\
\text { tamento total }\end{array}$ & 13,2 & 07,0 & $4,39-9,30$ \\
\hline \begin{tabular}{l} 
Desempenho acadêmico \\
\hline
\end{tabular} & 32,7 & 9,1 & $27,72-42,42$ \\
\hline
\end{tabular}

Nota: Dados referentes ao repertório de habilidades sociais e aos problemas de comportamento internalizantes $(\mathrm{N}=215)$.

Dados referentes aos problemas de comportamento externalizantes e total $(\mathrm{N}=190)$.

Dados referentes ao desempenho acadêmico $(\mathrm{N}=165)$.

Fonte: Dados da pesquisa.

Considerando a opinião das professoras, em três fatores, os alunos estavam com um repertório de habilidades sociais médio, segundo a amostra de referência (Bandeira, Del Prette \& Del Prette, \& Magalhães, 2009). Nos fatores Asserção positiva e 
Autocontrole, assim como o repertório de habilidades sociais total, as crianças e os adolescentes foram avaliadas com um repertório abaixo da média. Do total de alunos, $62,3 \%$ apresentaram repertório de habilidades sociais abaixo da média.

Dentre os comportamentos previstos no fator Asserção positiva, têm-se os de iniciar conversa, convidar para agrupar em atividades, apresentar a outras pessoas, fazer amigos, juntar-se a um grupo, questionar regras injustas e tecer comentários positivos sobre si (Bandeira et al., 2009). Tais comportamentos são importantes para o estabelecimento de vínculos com os pares e quanto às demandas acadêmicas, pois espera-se que um aluno consiga juntar-se a um grupo para fazer trabalho, que questione as regras da escola apropriadamente. Ao considerar que mais da metade da amostra está na $1^{\underline{a}}$ ou $2^{\underline{a}}$ séries do ensino fundamental, o fato de os alunos serem avaliados abaixo da média neste quesito vem a ser mais preocupante.

Isso porque ao ingressar no ensino fundamental novas tarefas são exigidas às crianças, como habilidades de interagir em grupo, fazer vínculo, se relacionar com crianças de sua idade e mais velhas, fazer as tarefas escolares no prazo determinado, cumprir todas as tarefas escolares, entre outras (Aspesi, Dessen \& Chagas, 2005).

Quanto ao Autocontrole, que se refere à habilidade de a criança controlar suas próprias emoções, como por exemplo, negociar conflitos, aceitar as ideias dos colegas, reagir apropriadamente a gozações, pressão e provocação dos colegas, pode-se inferir que tais crianças tendem a apresentar maior probabilidade de agredirem verbalmente ou fisicamente, de terem dificuldades de trabalhar em grupo, de resolverem os conflitos interpessoais de forma negativa e pouco construtiva. Aspectos que podem interferir negativamente no desempenho acadêmico, visto que crianças mais agressivas e hostis tendem a ser mais rejeitadas ou negligenciadas pelos pares (Bee, 2008).

Quanto aos problemas de comportamento, as crianças e os adolescentes foram avaliados pelas professoras como tendo problemas de comportamento internalizantes, problemas de comportamento externalizantes e problemas de comportamento total acima da média, segundo a amostra de referência. Por fim, quanto ao desempenho acadêmico, os alunos foram avaliados na média.

No que se refere à porcentagem de participantes com problemas de comportamento, 60,4\% foram avaliados como tendo problemas de comportamento total, $48,0 \%$ como tendo problemas de comportamento externalizantes e $49,0 \%$ como tendo problemas de comportamento internalizantes. Quanto ao desempenho acadêmico, 27,3\% dos participantes estavam abaixo da média.

Essa alta porcentagem de crianças e adolescentes apontada pelas professoras com problemas de comportamento é preocupante, pois os participantes desta amostra estão em idade escolar e podem estar em risco, visto que os problemas de comportamento estão relacionados ao baixo desempenho acadêmico, assim como ao desenvolvimento de problemas de conduta, abandono/evasão escolar na adolescência (Del Prette \& Del Prette, 2005; Gallo \& Williams, 2005).

No entanto, vale ressaltar que a avaliação das professoras está contextualizada ao ambiente escolar e ao de uma Organização Não Governamental, assim como tem-se a influência do caráter subjetivo dessa avaliação, uma vez que estudos mostram para uma variabilidade de classificação dos problemas de comportamento entre pais e professores ou mesmo quando se trata de crianças do sexo masculino e feminino (Bolsoni-Silva, Marturano, Pereira \& Manfrinato, 2006; Lópes, 2004; Marturano, Toller, \& Elias, 2005).

Além disso, um pouco menos de um terço da amostra apresentou baixo desempenho acadêmico. Crianças e adolescentes que vivenciam uma trajetória acadêmica pouco satisfatória tendem a apresentar problemas de motivação, acarretando mais problemas em seu desenvolvimento. Soma-se que essas crianças e adolescentes normalmente são encaminhadas para salas de recursos ou mesmo para atendimento psicológico (Kauffman, 2001).

Normalmente essas crianças e adolescentes têm problemas de autoestima, autoconceito, ou seja, os problemas de comportamento se relacionam com problemas emocionais (Del Prette \& Del Prette, 2005; Okano, Loureiro, Linhares \& Marturano, 2004; Stevenato, Loureiro, Linhares \& Marturano, 2003). Para Yunes e Szymanski (2001), os mecanismos de proteção ao desenvolvimento infantil estão relacionados com habilidades de assertividade, de resolução de problemas e elevada autoestima, aspectos estes considerados deficitários nos participantes desta amostra, ou seja, sem uma intervenção educativa tais crianças e adolescentes encontram-se em situação de risco ao desenvolvimento. 
A Tabela 2 mostra as correlações entre o repertório de habilidades sociais, os problemas de comportamento e o desempenho acadêmico das crianças e dos adolescentes, segundo a opinião das professoras.

Tabela 2 - Correlaç̃oes entre o repertório de habilidades sociais, os problemas de comportamento e o desempenho acadêmico das crianças e dos adolescentes

\begin{tabular}{lccccc}
\hline & $\begin{array}{c}\text { Habilidades sociais } \\
\text { (escore total) }\end{array}$ & PCE & PCI & PCT & DA \\
\hline F1-Responsabilidade/Cooperação & $0,521^{* * *}$ & $-0,687^{* * *}$ & $-0,374^{* * *}$ & $-0,700^{* * *}$ & $0,719^{* * *}$ \\
\hline F2-Asserção. & $0,453^{* * *}$ & $-0,474^{* * *}$ & $-0,348^{* * *}$ & $-0,509^{* * *}$ & $0,629^{* * *}$ \\
\hline F3-Autocontrole. & $0,449^{* * *}$ & $-0,715^{* * *}$ & $-0,265^{* * *}$ & $-0,698^{* * *}$ & $0,634^{* * *}$ \\
F4-Autodefesa. & $0,383^{* * *}$ & $-0,429^{* * *}$ & $-0,303^{* * *}$ & $-0,451^{* * *}$ & $0,567^{* * *}$ \\
F5-Cooperação com pares. & $0,368^{* * *}$ & $-0,504^{* * *}$ & $-0,166^{* *}$ & $-0,493^{* * *}$ & $0,518^{* * *}$ \\
$\begin{array}{l}\text { Problemas de comportamento } \\
\text { externalizantes - PCE }\end{array}$ & $-0,693^{* * *}$ & --- & $0,365^{* * *}$ & $0,957^{* * *}$ & $-0,656^{* * *}$ \\
$\begin{array}{l}\text { Problemas de comportamento } \\
\text { internalizantes - PCI }\end{array}$ & $-0,363^{* * *}$ & $0,365^{* * *}$ & --- & --- & $-0,409^{* * *}$ \\
$\begin{array}{l}\text { Problemas de comportamento } \\
\text { total - PCT }\end{array}$ & $-0,706^{* * *}$ & $0,957^{* * *}$ & $0,599^{* * *}$ & --- & $-0,655^{* * *}$ \\
\hline
\end{tabular}

Nota: ${ }^{*} \mathrm{p}<0,05 ;{ }^{* *} \mathrm{p}<0,01 ;{ }^{* * *} \mathrm{p}<0,001 . \mathrm{DA}=$ desempenho acadêmico.

Fonte: Dados da pesquisa.

Como mostram os dados da Tabela 2, o repertório de habilidades sociais (considerando os cinco fatores que compõem a escala e o repertório de habilidades sociais total) estava negativamente correlacionado com os problemas de comportamento externalizantes, internalizantes e total. Além disso, os problemas de comportamento internalizantes, externalizantes e total também estavam positivamente correlacionados entre si. Por fim, quanto maior o desempenho acadêmico, maior o repertório de habilidades sociais e menor os índices de problemas de comportamento das crianças e dos adolescentes. Esses resultados estão condizentes com dados da literatura nacional e internacional (Cia, Pamplin \& Del Prette, 2006; Del Prette \& Del Prette, 2005; Dunn, Cheng, O'Connor, \& Bridges, 2004). Tais relações vêm demonstrar que as escolas deveriam se atentar aos aspectos socioemocionais das crianças ao promover intervenções educativas para melhorar o desempenho acadêmico.

Por exemplo, para diminuir os comportamentos externalizantes dos alunos, existem medidas para as escolas, pais e crianças. Para as escolas, é necessária boa liderança, clima ordenado e não repressivo, participação dos professores nas decisões da escola, boa relação entre professores, grandes expectativas quanto à aprendizagem dos alunos e monitoramento frequente e com feedbacks às performances dos alunos. Quanto às interações entre professores e alunos com mais problemas de comportamento é importante que os professores comuniquem-se frequentemente com os alunos, peça a opinião deles, valorize os bons comportamentos, evite fazer ameaças e expor os maus comportamentos dos alunos para os demais, aplique regras e seja consistente nas consequências do seu cumprimento ou não. Com relação ao trabalho com os alunos, as escolas poderiam se beneficiar com treinamento de habilidades sociais (como empatia, expressão de sentimentos, assertividade, entre outras habilidades), oferecer modelos de comportamentos adequados às crianças, discutir com os alunos as diferenças entre as pessoas e o respeito, por meio de fóruns, debates, discussão de filmes, etc. (Stelko-Pereira \& Williams, 2009; Lópes, 2004).

Somado a isso, seria desejável planejar atividades para aumentar o interesse do aluno em frequentar a escola, visto que o estudo de Gallo e Williams (2008) mostrou que, segundo os adolescentes que cometeram algum ato infracional, a escola não tem atrativo algum para eles. 


\section{Considerações finais}

0 presente estudo mostrou uma correlação negativa entre o repertório de habilidades sociais, 0 desempenho acadêmico e os problemas de comportamento das crianças e adolescentes e uma correlação positiva entre o desempenho acadêmico e o repertório de habilidades sociais. Tais resultados são indicativos de que essas correlações estejam presentes ao longo do ensino fundamental, verificando-se a importância de as escolas e pais se atentarem a esses fatores em diferentes faixas etárias.

Nota-se a necessidade de intervenções de caráter de prevenção secundária e/ou terciária, para minimizar a incidência e intensidade de problemas de comportamento, pois tais crianças e adolescentes estão em risco de fracasso acadêmico, envolvimento em atividades ilícitas, evasão escolar, dentre outros. Como medidas interventivas têm-se: treino de habilidades sociais positivas com pais, professores e demais agentes educativos para que sejam modelos de comportamento das crianças; treino com os professores para estabelecerem contingências comportamentais diferenciadas aos comportamentos dos alunos (Costa, Williams \& Costa, no prelo); desenvolver com os professores habilidades de escutar e compreender os alunos com problemas de comportamento (que envolve sinceridade, humildade, paciência, cordialidade, oportunidade, discrição e coragem); proporcionar à criança o desenvolvimento de um autoconceito positivo (González, 2007); trabalhar preventivamente com os pais (Cia, Barham \& Fontaine, 2010; Gonzáles, 2007).

Lópes (2004) ainda complementa que as escolas deveriam estabelecer normas claras de conduta e sistema de controle para auxiliar no aprendizado de habilidades sociais das crianças, com os seguintes princípios:

a) origem consensual ou refletida das normas, tanto pelos alunos, quanto pelos agentes educativos;

b) explicar a importância das normas para o bem-estar social e individual;

c) possibilidade de discussão e de mudanças das normas;

d) manter a coerência, exigir seu cumprimento e prever as consequências que serão estabelecidas se as normas forem ou não cumpridas; e) prever estratégias para resolver conflitos que ultrapassem o próprio educador, como treinamento de habilidades sociais com as crianças, em contexto de sala de aula.

Ressalta-se que os dados deste estudo devem ser vistos com cautela, visto que se trata de um estudo correlacional, em que não se pode estabelecer relações de causa e efeito entre as variáveis estudadas (Cozby, 2006). Além disso, apesar de ser significativa, a amostra é reduzida a determinadas escolas de um município e a de uma Organização Não Governamental. Futuros estudos são indicados, tanto longitudinais (para acompanhar a trajetória do desenvolvimento das crianças com problemas de comportamento), quanto de pesquisa-intervenção para verificar a eficácia de intervir com pais, professores e próprias crianças/adolescentes inseridos no ensino fundamental sobre os problemas de comportamento dos mesmos.

\section{Referências}

Ablow, J. C., Measelle, J. R., Cowan, P. A., \& Cowan, C. P. (2009). Linking marital conflict and children's adjustment: The role of young children's perceptions. Journal of Family Psychology, 23(4), 485-499.

Achenbach, T. M. (1991). Manual of the child behavior checlist/ 4-18 and 1991 profile. Burlington: University of Vermont, Departament of Psychiatry.

Achencach, T. M., \& Edelbrock, C. S. (1978). The classification of child psychopathology: A review and analysis of empirical efforts. Psycological Bulletin, 85(6), 1275-1301.

Aspesi, C. C., Dessen, M. A., \& Chagas, J. F. (2005). A ciência do desenvolvimento humano: Uma perspectiva interdisciplinar. In M. A. Dessen \& A. L. S. Costa (Org.). A ciência do desenvolvimento humano: Tendências atuais e perspectivas futuras (pp. 19-36). Porto Alegre: Artmed.

Bandeira, M., Del Prette, Z. A. P., Del Prette, A., \& Magalhães, T. (2009). Validação das escalas de habilidades sociais, Comportamentos problemáticos e competência acadêmica (SSRS-BR) no ensino fundamental. Psicologia: Teoria e Pesquisa, 25(2), 271-282. 
Bandeira, M., Roch, S. S., Souza, T. M. P., Del Prette, Z. A. P., \& Del Prette, A. (2006). Comportamentos problemáticos em estudantes do ensino fundamental: Características da ocorrência e relação com habilidades sociais e dificuldades de aprendizagem. Estudos de Psicologia, 11(2), 199-208.

Bee, H. (2008). A criança em desenvolvimento. Porto Alegre: Artmed, 2008.

Bolsoni-Silva, A. T., Marturano, E. M., Pereira, V. A., \& Manfrinato, J. W. S. (2006). Habilidades sociais e problemas de comportamento em pré-escolares: Comparando avaliações de mães e de professoras. Psicologia: Reflexão e Crítica, 19(3), 430-436.

Brancalhone, P. G., Fogo, J. C., \& Williams, L. C. A. (2004). Crianças expostas a violência conjugal: Avaliação do desempenho acadêmico. Psicologia: Teoria e Pesquisa, 20(2), 113-117.

Brasil. (2002). Estratégias e orientações para a educação de alunos com dificuldades de aprendizagem associadas às condutas típicas. Brasília: MEC; INEP.

Brasil. (2006). Dados da Educação Especial no Brasil. Brasília: MEC; INEP.

Cia, F., Barham, E. J., \& Fontaine, A. M. G. V. (2010). Impactos de uma intervenção com pais: Desempenho acadêmico e comportamento das crianças na escola. Psicologia: Reflexão e Crítica, 23(3), 43-53.

Cia, F., Pamplin, R. C. O., \& Del Prette, Z. A. P. (2006). Comunicação e participação pais-filhos: Correlação com habilidades sociais e problemas de comportamento dos filhos. Paidéia: Cadernos de Psicologia e Educação, 6(35), 395-406.

Costa, C. S. L., Williams, L. C. A., \& Cia, F. (no prelo). Efeitos de uma intervenção com monitores de Organização Não Governamental para minimizar problemas de comportamento em crianças. Psicologia: Reflexão e Crítica.

Cozby, P. C. (2006). Métodos de pesquisa em ciências do comportamento. São Paulo: Atlas.

D’Avila-Bacarji, K. M. G., Marturano, E. M., \& Elias, L. C. S. (2005). Suporte parental: Um estudo sobre crianças com queixas escolares. Psicologia em Estudo, 10(1), 107-115.

Del Prette, Z. A. P., \& Del Prette, A. (1999). Psicologia das habilidades sociais: Terapia e educação. Petrópolis: Vozes.
Del Prette, A. \& Del Prette, Z. A. P. (2001). Psicologia das relações interpessoais: Vivências para o trabalho em grupo. Petrópolis: Vozes.

Del Prette, Z. A. P., \& Del Prette, A. (2005). Psicologia das habilidades sociais na infância: Teoria e Prática. Petrópolis: Vozes.

Dessen, M. A., \& Costa, A. L. (2005). A ciência do desenvolvimento humano. Porto Alegre: Artmed.

Dessen, M. A., \& Szelbracikowski, A. C. (2004). Crianças com problemas de comportamento exteriorizado e a dinâmica familiar. Interação em Psicologia, 8(2), 171-180.

Dunn, J., Cheng, H., O’Connor, T. G., \& Bridges, L. (2004). Children's perspective on their relationships with their nonresident fathers: Influences, outcomes and implications. Journal of Child Psychology and Psychiatry, 45(3), 553-566.

Gallo, A. E., \& Williams, L. C. A. (2005). Adolescentes em conflito com a lei: Uma revisão dos fatores de risco para a conduta infracional. Psicologia: Teoria e Prática, 7(1), 81-95.

Gallo, A. E., \& Williams, L. C. A. (2008). A escola como fator de proteção à conduta infracional de adolescentes. Cadernos de Pesquisa, 38(133), 51-59.

Gomide, P. I. C. (2004). Pais presentes pais ausentes: Regras e limites. Petrópolis: Vozes.

González, E. (2007). Problemas de conduta: Conceito e intervenção psicoeducacional. In E. González (Org.). Necessidades educacionais específicas: Intervenção psicoeducacional (pp. 270-294). Porto Alegre: Artmed.

Gresham, F. M., \& Elliott, S. N. (1990). Social skills rating system. Circle Pines, MN: American Guidance Service, Inc.

Graminha, S. S. V. (1998). Recursos metodológicos para pesquisas sobre riscos e problemas emocionais e comportamentais na infância. In G. Romanelli \& Z. M. Biasoli-Alves (Org.). Diálogos metodológicos sobre práticas de pesquisa (pp. 71-86). Ribeirão Preto: Legis Summa.

Hinshaw, S. P. (1992). Externalizing behavior proble$\mathrm{ms}$ and academic underachievement in childhood and adolescence: Causal relationships and underlying mechanisms. Psychological Bulletin, 111(1), 127-155. 
Kauffman, J. M. (2001). Characteristics of emotional and behavioral disorders of children and youth. Upper Saddle River: Merril Prentice Hall.

Kazdin, A. E. (1987). Treatment of antisocial behavior in children: Current status and future directions. Psychological Bulletin, 102(2), 187-203.

Kazdin, A. E. (1996). Problem solving and parent management in treating aggressive and an antisocial behavior. In E.D. Hibbs \& P.S. Jensen (Org.). Psychosocial treatments for child and adolescent disorders: Empirically based strategies for clinical practice (pp. 377-408). Washington: American Psychological Association.

Kazdin, A. E., \& Whitley, M. K. (2003). Treatment of parental stress to enhance therapeutic of parental stress to enhance therapeutic change among children referred for aggressive and antisocial behavior. Journal of Consulting and Clinical Psychology, 71(3), 504-515.

Lindsey, E. W., Caldera, Y. M., \& Iankersley, L. (2009). Marital conflict and the quality of young children's peer play behavior: The mediating and moderating role of parent-child emotional reciprocity and attachment security. Journal of Family Psychology, 23(2), 130-145.

Lopes, F. (2004). Problemas afetivos e de conduta em sala de aula. In C. Coll, A. Marchesi \& J. Palácios (Org.). Desenvolvimento psicológico e educação 3: Transtornos do desenvolvimento e necessidades educativas especiais (pp. 113-128). Porto Alegre: Artmed, 2004.

Marinho, M. L. (2003). Comportamento antissocial infantil: Questões teóricas e de pesquisa. In A. Del Prette \& Z. A. P. Del Prette (Org.). Habilidades sociais, desenvolvimento e aprendizagem: Questões conceituais, avaliação e intervenção. Campinas: Alínea.

Marturano, E. M., Linhares, A. B. M., \& Loureiro, S. R. (2004). Vulnerabilidade e proteção: Indicadores na trajetória de desenvolvimento do escolar. São Paulo: Casa do Psicólogo.

Marturano, E. M., Toller, G. P., \& Elias, L. C. S. (2005). Gênero, adversidade e problemas socioemocionais associados à queixa escolar. Estudos de Psicologia, 22(4), 371-380.
Okano, C. B., Loureiro, S. R., Linhares, M. B. M., \& Marturano, E. M. (2004). Crianças com dificuldades escolares atendidas em programa de suporte psicopedagógico na escola: Avaliação do autoconceito. Psicologia: Reflexão e Crítica, 17(1), 121-128.

Pacheco, J., Alvarenga, P., Reppold, C., Piccinini, C. A., \& Hutz, C. (2005). Estabilidade do comportamento antissocial na transição da infância para a adolescência: Uma perspectiva desenvolvimentista. Psicologia: Reflexão e Crítica, 18(1), 55-61.

Parke, R. D. (2004). Development in the family. Annual Reviews Psychology, 55, 365-399.

Patterson, G. R., Debaryshe, B. D., \& Ramsey, E. (1989). A developmental perspective on anti-social behavior. American Psychologist, 44(2), 329-335.

Patterson, G. R., Reid, J. B., \& Dishion, T. J. (1992). Antisocial boys. Eugene: Castalia, 1992.

Santos, P. L., \& Graminha, S. S. V. (2006). Problemas emocionais e comportamentais associados ao baixo rendimento acadêmico. Estudos de Psicologia, 11(1), 71-86.

Stelko-Pereira, K., \& Williams, L. C. A. (2009). Ações para prevenção de violência escolar. In L. C. A. Williams, R. C. Padovani, E. A. C. Araújo, A. C. S. Pereira, G. R. 0 . Eisentein (Org.). Fortalecendo a rede de proteção da criança e do adolescente (pp. 66-67). São Carlos: LAPREV.

Stevanato, I. S., Loureiro, S. R., Linhares, M. B. M., \& Marturano, E. M. (2003). Autoconceito de crianças com dificuldades de aprendizagem e problemas de comportamento. Psicologia em Estudo, 8(1), 67-76.

Vila, E. M. (2005). Treinamento de habilidades sociais em grupo com professores de crianças com dificuldades de aprendizagem: Uma análise sobre procedimentos e efeitos da intervenção. Dissertação de Mestrado Universidade Federal de São Carlos, São Carlos, São Paulo.

Walker, H. M., \& Sprague, J. R. (1999). The path to school failure, delinguency, and violence: Causalfactors and some potential solutions. Interventions in School and Clinic, 35, 67-73.

Yunes, M. A., \& Szymanski, H. (2001). Resiliência: Noção, conceitos afins e considerações críticas. In J. Tavares (Org.). Resiliência e Educação (pp. 13-42). São Paulo: Cortez. 